\title{
Criminologie
}

\section{Les conséquences développementales de la maltraitance des filles}

\section{Carolyn A. Smith et Timothy O. Ireland}

Volume 38, numéro 1, printemps 2005

Filles et déviance : perspectives développementales

URI : https://id.erudit.org/iderudit/011486ar

DOI : https://doi.org/10.7202/011486ar

Aller au sommaire du numéro

\section{Éditeur(s)}

Les Presses de l'Université de Montréal

ISSN

0316-0041 (imprimé)

1492-1367 (numérique)

Découvrir la revue

Citer cet article

Smith, C. A. \& Ireland, T. O. (2005). Les conséquences développementales de la maltraitance des filles. Criminologie, 38(1), 67-102.

https://doi.org/10.7202/011486ar
Résumé de l'article

Objet : L'étude traite des conséquences des mauvais traitements, corroborés par des instances officielles, sur le comportement antisocial et sur d'autres indicateurs connexes chez les femmes d'un échantillon longitudinal et représentatif de la ville de Rochester aux États-Unis.

Méthodologie : Les données proviennent de l'étude de Rochester sur le développement des jeunes, une étude de cohortes portant sur l'apparition de problèmes comportementaux à partir d'un échantillon de 1000 jeunes suivis de l'âge de 13 ans jusqu'à l'âge adulte. Les analyses se concentrent uniquement sur les 271 adolescentes d'origine afro-américaine ou hispanique de l'échantillon. La consultation des registres du Service de protection de l'enfance du comté a permis de découvrir 74 (27\%) filles ayant subi des mauvais traitements. Les conséquences qui seront analysées sont les arrestations, la délinquance et la violence autorapportées, la consommation de drogues, les problèmes liés à la consommation d'alcool, la violence subie et manifestée dans les relations de couple, les symptômes dépressifs et les risques de contracter des maladies transmises sexuellement (MTS) et le SIDA au début de l'âge adulte (20-22 ans).

Résultats : Les analyses bivariées indiquent que la maltraitance est associée à plusieurs conséquences négatives au début de l'âge adulte. La régression logistique, qui contrôle les variables sociodémographiques et la délinquance juvénile, confirme que la maltraitance augmente les risques d'apparition de plusieurs conséquences négatives. Les résultats suggèrent également que l'abus sexuel peut avoir des répercussions particulièrement graves chez les jeunes femmes.

Implications : La présente recherche vise l'amélioration des programmes de prévention et de traitement pour les jeunes femmes ayant été abusées et négligées au cours de l'enfance.
Ce document est protégé par la loi sur le droit d'auteur. L'utilisation des services d'Érudit (y compris la reproduction) est assujettie à sa politique d'utilisation que vous pouvez consulter en ligne.

https://apropos.erudit.org/fr/usagers/politique-dutilisation/ 


\title{
Les conséquences développementales de la maltraitance des filles ${ }^{1}$
}

\author{
Carolyn A. Smith \\ School of Social Welfare \\ Université d'Albany \\ csmith@albany.edu \\ Timothy O. Ireland \\ Département de justice criminelle et de criminologie \\ Université Niagara \\ toi@niagara.edu
}

RÉSUMÉ • Objet: L'étude traite des conséquences des mauvais traitements, corroborés par des instances officielles, sur le comportement antisocial et sur d'autres indicateurs connexes chez les femmes d'un échantillon longitudinal et représentatif de la ville de Rochester aux États-Unis.

Méthodologie: Les données proviennent de l'étude de Rochester sur le développement des jeunes, une étude de cohortes portant sur l'apparition de problèmes comportementaux à partir d'un échantillon de 1000 jeunes suivis de l'âge de 13 ans jusqu'à l'âge adulte. Les analyses se concentrent uniquement sur les 271 adolescentes d'origine afro-américaine ou hispanique de l'échantillon. La consultation des registres du Service de protection de l'enfance du comté a permis de découvrir 74 (27\%) filles ayant subi des mauvais traitements. Les conséquences qui seront analysées sont les arrestations, la délinquance et la violence autorapportées, la consommation de drogues, les problèmes liés à la consommation d'alcool, la violence subie et manifestée dans les relations de couple, les symptômes dépressifs et les risques de contracter des maladies transmises sexuellement (MTS) et le SIDA au début de l'âge adulte (20-22 ans).

1. Cet article a été produit grâce aux subventions suivantes: 86-JN-CX-0007 (S-3) octroyée par l'Office of Juvenile Justice and Delinquency Prevention, Office of Justice Programs, U.S. Department of Justice; 5 R01 DA05512-02 du National Institute on Drug Abuse et SBR09123299 de la National Science Foundation. Ce projet de recherche a également été rendu possible grâce aux subventions NICHD (P30 HD32041) et NSF (SBR-9512290) accordées au Center for Social and Demographic Analysis at the University at Albany. Les points de vue ou les opinions exprimés dans le cadre de cette étude sont ceux des auteurs et ne représentent pas nécessairement les positions ou les politiques des organismes subventionnaires. Texte traduit par C. Pilon et révisé par N. Lanctôt. 
Résultats: Les analyses bivariées indiquent que la maltraitance est associée à plusieurs conséquences négatives au début de l'âge adulte. La régression logistique, qui contrôle les variables sociodémographiques et la délinquance juvénile, confirme que la maltraitance augmente les risques d'apparition de plusieurs conséquences négatives. Les résultats suggèrent également que l'abus sexuel peut avoir des répercussions particulièrement graves chez les jeunes femmes.

Implications: La présente recherche vise l'amélioration des programmes de prévention et de traitement pour les jeunes femmes ayant été abusées et négligées au cours de l'enfance.

ABSTRACT - Purpose: The current study investigates the impact of substantiated maltreatment on antisocial behavior and other outcomes among young adult women in a representative community sample followed longitudinally.

Methods: Data come from the Rochester Youth Development Study (RYDS), a cohort study of the development of problem behaviors in a sample of 1,000 urban youth followed from age 13 into adulthood. Subjects include 68\% African American, 17\% Hispanic, and 15\% White youth. This analysis focuses only on the 271 young women in the panel. County Child Protective Service records were searched and 74 (27\%) of the young women experienced substantiated maltreatment. Outcomes include arrest, self-reported general and violent offending, drug use, problem alcohol use, partner violence perpetration and victimization, depressive symptoms, and STD/HIV risk in early adulthood (ages 20-22).

Results: We find that experiencing substantiated maltreatment increases the risk of most outcomes at the bivariate level. Employing logistic regression, maltreatment increases the odds of several negative consequences after controlling for confounding sociodemographic variables including poverty, family structure, parental education, race/ethnicity, and adolescent delinquency. Results also suggest that sex abuse may be particularly problematic for these young women in emerging adulthood.

Implications: We discuss implications for understanding pathways and processes, and for improving prevention and treatment for abused and neglected young women.

\section{Introduction}

La récente attention portée à la délinquance et aux comportements antisociaux des femmes s'est longuement fait attendre, la recherche empirique et théorique s'étant surtout concentrée sur les comportements masculins au cours des dernières décennies (Chesney-Lind et Shelden, 1998; Hoyt et Scherer, 1998; Keenan et al., 1999). Néanmoins, un des domaines de la recherche qui s'est immanquablement attardé aux femmes concerne les conséquences développementales des mauvais traitements durant l'enfance. De manière générale, les mauvais traitements sont associés à une série de conséquences développementales problématiques, telles la délinquance et la violence, la violence conjugale, la consommation de drogues ainsi que l'intériorisation de problèmes 
comme les symptômes dépressifs (Ireland et Widom, 1994; Smith et Thornberry, 1995 ; Kakar, 1996 ; Kaplan et al., 1999 ; Lemmon, 1999; Thornberry et al., 2001; Elliott et al., 2002). Malgré tout, rares sont les recherches qui se sont attardées aux conséquences à long terme des mauvais traitements infligés aux filles en recourant à des études prospectives menées auprès d'échantillons puisés dans la collectivité selon des critères précis.

Plusieurs raisons démontrent la nécessité d'examiner plus systématiquement les conséquences des mauvais traitements infligés aux filles. Premièrement, puisque les filles et les garçons sont susceptibles d'être exposés à des formes différentes de mauvais traitements, leurs réactions à ces mauvais traitements peuvent varier. Bien que les statistiques nationales indiquent des taux similaires d'exposition à la négligence et à la violence physique (USDHHS, 2004), les recherches démontrent invariablement que les filles sont plus victimes d'abus sexuel que les garçons au cours de l'enfance (Putnam, 2003). Cet état de fait a conduit, par conséquent, bon nombre de chercheurs à étudier spécifiquement les conséquences de l'abus sexuel des filles (Kendler et al., 2000; Buzi et al., 2003 ; Dunlap et al., 2003; Merrill et al., 2003 ; Simpson, 2003; Chen et al., 2004; Roberts et al., 2004). Il importe toutefois de mentionner que l'abus sexuel durant l'enfance se produit rarement de façon isolée, d'autres formes de mauvais traitements y étant souvent jumelées (Bagley et Mallick, 2000; Spohn, 2000; Thornberry et al., 2001).

Deuxièmement, même lorsque confrontés à des facteurs de risque similaires, les réactions des filles et des garçons tendent à différer. En effet, si les réactions des filles se traduisent le plus souvent par des comportements intériorisés tels que la dépression, du côté des garçons, ce sont les comportements agressifs et antisociaux qui prédominent (Widom, 1989a; Moffitt et al., 2001). Considérant cette différence entre les sexes, Rutter et al. (2003) émettent l'hypothèse selon laquelle la force de la relation qui unit les mauvais traitements à la délinquance ne serait pas aussi élevée, voire même serait complètement absente, dans des échantillons uniquement composés de filles en comparaison aux échantillons mixtes ou masculins (Chandy et al., 1996; Bergen et al., 2004). Les recherches demeurent toutefois ambiguës sur cette question, le lien entre les conduites antisociales des filles et leur victimisation antérieure n'étant pas encore clairement démontré (Widom, 1989b; Herrera et McCloskey, 2001; Widom et Maxfield, 2001; Wolfe et al., 2001). De la même façon, d'autres conséquences résultant de la 
maltraitance durant l'enfance, comme l'alcoolisme, la dépression, les comportements sexuels à risque (Elliott et al., 2002; Simpson et Miller, 2002; Thompson et al., 2004), ont également fait l'objet de peu d'études prospectives. Ce faisant, force est de constater que beaucoup de travail reste encore à faire pour connaitre l'étendue des conséquences à long terme des mauvais traitements dont sont victimes les filles.

Troisièmement, les filles qui sont maltraitées durant l'enfance sont susceptibles d'être l'objet de mesures judiciaires disproportionnées comparativement aux garçons. À titre d'exemple, McClellan et al. (1997) relèvent que $26 \%$ des femmes détenues interviewées dans le cadre de leur recherche rapportaient avoir été victimes d'abus ou de mauvais traitements sexuels durant l'enfance, alors que chez les hommes détenus, cette proportion n'atteignait que 4,5\% (voir également le Bureau of Justice Statistics, 1994; Daly, 1994). Ces disparités entre les sexes suggèrent que les femmes qui ont été maltraitées empruntent, plus que les hommes maltraités, des trajectoires qui les mènent vers l'incarcération. Dans la même veine, les femmes ayant séjourné dans des centres de désintoxication, souvent à la suite d'une exigence de la Cour, déclarent également de hauts taux de victimisation sexuelle durant l'enfance (Kang et al., 1999). Bien qu'intéressantes, la plupart des études qui abordent la maltraitance sont de nature rétrospective et s'appuient sur des populations institutionnalisées; leurs résultats sont donc difficiles à généraliser à l'ensemble de la population.

Ces constatations ont amené les criminologues à débattre plus profondément des similitudes et des différences entre les sexes sur le plan des trajectoires délinquantes et criminelles (Rutter et al., 2003). Il est possible que les trajectoires liant les mauvais traitements et les comportements antisociaux different chez les filles, en raison de leurs plus hauts taux de victimisation sexuelle et des traumatismes qui en découlent (Cicchetti et Toth, 1997). Malgré tout, tant et aussi longtemps que l'étendue des conséquences de la maltraitance ne sera pas mesurée, l'exploration des trajectoires qui y sont associées demeure un exercice prématuré.

\section{Recension des écrits}

La maltraitance des enfants constitue le terme généralement utilisé pour décrire toutes les formes d'abus et de négligence qui surviennent à la petite enfance, l'enfance ou l'adolescence. Bien qu'il n'existe pas de 
définition universellement reconnue de ce phénomène, le gouvernement fédéral américain définit l'abus et la négligence des enfants comme:

the physical and mental injury, child sexual abuse, negligent treatment, or maltreatment of a child under the age of 18 by a person who is responsible for the child's welfare under circumstances which indicate that the child's health or welfare is harmed or threatened (Child Abuse Prevention and Treatment Act, CAPTA 42 U.S.C.A. 5106g).

Étendue du problème: Les données nationales américaines indiquent que la proportion des garçons et des filles qui sont victimes de maltraitance est équivalente. En 2002, 48,1\% des enfants maltraités étaient des garçons et 51,9\% étaient des filles (USDHHS, 2004). Alors que la plupart des formes de maltraitance se répartissent également entre les sexes, seul l'abus sexuel se démarque en étant quatre fois plus fréquent chez les filles que chez les garçons. Malgré cette différence, la coexistence de différents types de maltraitance s'observe tant chez les garçons que chez les filles (Crittenden et al., 1994; Manly et al., 1994; McCloskey et al., 1995; McGee et al., 1997). Ce faisant, l'abus sexuel - lequel a souvent été étudié de façon isolée - se produit souvent simultanément à d'autres types de mauvais traitements (Hughes et al., 1989; Bagley et Mallick, 2000; Thornberry et al., 2001). À titre d'exemple, l'étude de Bolger et Patterson (2001), qui s'appuie sur un échantillon de 107 participants ayant subi des mauvais traitements, démontre que $52 \%$ de ces victimes ont expérimenté plusieurs formes de maltraitance au cours de leur enfance. Dans la même veine, Edwards et al. (2003) ont amassé des données sur plus de 8000 sujets et concluent que parmi les femmes ayant rapporté avoir été victimes d'abus sexuel à l'enfance $(25,1 \%)$, plus de la moitié rapportaient également au moins une autre forme d'abus à cette même période.

Perspective conceptuelle: La psychopathologie développementale ainsi que bon nombre de théories, incluant celle de l'apprentissage social, de l'adversité, de l'attachement et du traumatisme, conçoivent que des perturbations développementales découlent des mauvais traitements subis durant l'enfance (Cicchetti et al., 1993 ; Crick et Dodge, 1994; Simons et al., 1995; Widom, 2000a). Considérant l'étendue des mécanismes susceptibles de perturber le développement d'un enfant, il n'est pas étonnant de constater que les conséquences des mauvais traitements peuvent compromettre le développement comportemental, émotif, 
cognitif et social de l'enfant (Kolbo et al., 1996; Dodge et al., 1997; Graham-Bermann et Levendosky, 1998; Osofsky, 1999; Rossman, 2001). Le passage de l'adolescence à l'âge adulte peut être particulièrement critique, puisqu'il représente une période au cours de laquelle l'indépendance à l'égard des rôles sociaux et des attentes normatives est recherchée (Arnett, 2000). Les mauvais traitements peuvent alors entraîner une cascade de conséquences à l'aube de l'âge adulte, à un point tel que certains adolescents en viennent à emprunter une trajectoire de vie qui perturbe le développement de leurs compétences sociales et les entraîne dans une série de problèmes comportementaux (Newcomb et Bentler, 1988; Furstenberg, 1990; Sampson et Laub, 1993; Kandel et al., 1995 ; Locke et Newcomb, 2004 ; Thornberry et Krohn, sous presse).

Bien que le présent article ne cherche pas spécifiquement à tester des hypothèses en compétition, il clarifiera les connaissances quant aux conséquences négatives de la maltraitance des filles, une fois que ces dernières atteignent l'âge adulte. Une telle entreprise s'avère particulièrement importante, puisque plusieurs victimes de mauvais traitements ne semblent pas en subir des répercussions à long terme (Ireland et al., 2002). Néanmoins, rappelons que la plupart des recherches effectuées sur la maltraitance des filles reposent sur des études rétrospectives et sur des échantillons non représentatifs. Dans la recension des écrits, nous avons sélectionné, autant que possible, les études qui ne comportent pas les lacunes méthodologiques repérées par d'autres chercheurs (spécialement par Widom, 1989a). Ce faisant, notre recension s'est particulièrement intéressée aux études qui ont eu recours à de grands échantillons ou à des échantillons représentatifs de femmes, à des devis de recherche de nature prospective, ainsi qu'à des mesures rigoureuses de la maltraitance et de ses conséquences.

Délinquance et violence: La littérature indique que le fait d'avoir subi des mauvais traitements durant l'enfance constitue un important facteur de risque de la délinquance et de la violence des filles. Ainsi, les filles maltraitées sont plus susceptibles d'être arrêtées que leurs consœurs qui n'ont jamais été maltraitées (Widom, 1989b; Spohn, 2000 ; HollisterWagner et al., 2001; Widom et Maxfield, 2001; Wolfe et al., 2001; Herrera et McCloskey, 2003; Siegel et Williams, 2003; Bergen et al., 2004). Widom et Maxfield (2001) mettent en lumière une relation évidente entre la maltraitance des filles et leurs probabilités d'être arrêtées pour des gestes de violence au début de l'âge adulte, les probabilités observées étant respectivement de $8,2 \%$ et de 3,6\% ( $p<0,05)$ pour 
les filles maltraitées et les filles non maltraitées. Siegel et Williams (2003) constatent aussi que les femmes victimes d'abus sexuel à l'enfance sont significativement plus susceptibles de se faire arrêter à l'âge adulte comparativement à un groupe témoin. Alors que certaines études indiquent que l'abus sexuel constitue un puissant facteur menant les filles à des trajectoires criminogènes (Herrera et McCloskey, 2003; Siegel et Williams, 2003), d'autres soulignent que l'abus physique et la négligence perturbent tout autant le développement des filles (Spohn, 2000; Herrera et McCloskey, 2001; Hollister-Wagner et al., 2001).

Violence $d u$ partenaire: Plusieurs études rétrospectives démontrent que la maltraitance à l'enfance constitue un facteur de risque reconnu de la violence conjugale, particulièrement chez les jeunes couples, mais aussi chez les femmes (ex., Banyard et al., 2000; Stith et al., 2000; Wolfe et al., 2001). Il appert toutefois que cette problématique a rarement été étudiée à partir d'études prospectives (Riggs et al., 1990; Follingstad et al., 1991; Foo et Margolin, 1995; Wolfe et al., 1998). L'étude de White et Widom (2003) est une des seules études prospectives dans ce domaine. Elle indique que les filles ayant été maltraitées, tout comme les garçons maltraités, sont plus enclines à frapper ou à lancer des objets à leur partenaire. Cependant, les mécanismes differrent selon le sexe. White et Widom (2003) considèrent quatre effets médiateurs possibles: l'agression en bas âge, le trouble de la personnalité antisociale, les problèmes liés à l'alcool et l'hostilité. Chez les garçons, le trouble de la personnalité antisociale constitue la phase intermédiaire entre la maltraitance durant l'enfance et la violence ultérieure dans les relations de couple. Du côté des filles, les problèmes de cette période transitoire sont la personnalité antisociale, les problèmes d'alcool et l'hostilité.

Dépression et symptômes dépressifs: La maltraitance est également associée aux troubles affectifs. La dépression et le comportement suicidaire sont depuis fort longtemps associés à la maltraitance durant l'enfance (Toth et al., 1992; Langinrichsen-Rohling et al., 1998; Kaplan et al., 1998, 1999; Salzinger, 1999; Rossman, 2001). Des études portant sur les femmes souffrant de dépression révèlent de façon constante leur forte exposition à la maltraitance durant l'enfance (Briere, 1992; Brown et al., 1999; Kendler et al., 2000; Putnam, 2003). D'autres types de maltraitance ont aussi été associés à la dépression (Gutierres et Todd, 1997). Ce lien n'est toutefois pas clairement établi, puisque Mannarino et al. (1991) ont observé, lors d'un suivi auprès de filles ayant été abusées sexuellement, que les tendances dépressives autorévélées par ces 
dernières ne different pas de celles rapportées par un groupe témoin n'ayant pas été maltraité. Des études longitudinales ont aussi mis en évidence la relation entre la maltraitance et la dysthymie, la dépression, les comportements suicidaires et d'autres symptômes intériorisés (Silverman et al., 1996; Bagley et Mallick, 2000; Horwitz et al., 2001; Thornberry et al., 2001; McCloskey et Lichter, 2003). Néanmoins, après avoir contrôlé les variables sociodémographiques et les données relatives à certains événements de la vie tels que le chômage, l'itinérance, le divorce, l'arrestation et les problèmes d'alcool ou de drogues d'un membre de la famille, Horwitz et al. (2001) n'observent pas de relation entre la maltraitance et les symptômes de dysthymie, et ce, tant chez les garçons que chez les filles. Toutefois, au-delà des symptômes, le diagnostic de dysthymie est quant à lui associé à la maltraitance antérieure des hommes.

Abus de psychotropes: La consommation de drogues et d'alcool a été associée à la maltraitance dans plusieurs études prospectives (Dembo et al., 1990; Ireland et Widom, 1994; Widom et al., 1995 ; Egeland, 1997 ; Thornberry et al., 2001 ; Smith et al., 2004). Cependant, peu d'études ont effectué une analyse différenciée selon le sexe. En s'appuyant sur un échantillon de jeunes en détention, Dembo et ses collègues (1990) indiquent que les participants maltraités à l'enfance rapportaient, à 15 ans, une consommation de drogues significativement plus importante que ceux qui révélaient ne pas avoir été maltraités. Leur consommation s'est aussi amplifiée plus que celle des non maltraités au cours de l'année suivante. Dembo et al. (1990) précisent que ces résultats ne variaient pas selon le sexe. Des résultats différents ressortent cependant de l'étude de Ireland et Widom (1994), laquelle est de nature prospective et contrôle statistiquement les contacts antérieurs avec le système de justice juvénile. Cette étude rapporte que la maltraitance subie avant 12 ans augmente le risque d'être arrêté pour un délit lié à l'alcool ou à la drogue à l'âge adulte, et ce, uniquement pour les femmes. Widom et White (1997) constatent aussi des effets plus marqués pour les filles : la maltraitance augmente le risque de dépendance ou de consommation abusive d'alcool et de drogues chez les filles, mais non chez les garçons. Ces résultats ne se reproduisent toutefois pas lorsque les enregistrements officiels de la maltraitance sont mis en relation avec un diagnostic d'abus ou de dépendance aux drogues selon le DSM-III-R (Widom et al., 1999).

Les problèmes liés spécifiquement à la consommation d'alcool semblent également être associés à la maltraitance des femmes dans bon 
nombre d'études prospectives (Widom et Hiller-Sturmhofel, 2001). Recourant à des données longitudinales, Dembo et al. (1990) observent que la victimisation sexuelle et physique rapportée par les participants est directement et indirectement liée à une consommation d'alcool ultérieure; ces relations étant les mêmes peu importe le sexe des participants. Toutefois, Widom et al. (1995) notent chez les femmes maltraitées, mais pas chez les hommes maltraités, une relation significative entre les mauvais traitements durant l'enfance et des symptômes récurrents reliés à l'alcool.

Comportements sexuels à risque: Le lien entre la maltraitance, particulièrement à l'enfance, et les comportements sexuels à risque chez les femmes est bien établi, bien que la plupart des études qui établissent ce lien sont de nature rétrospective (Kendall-Tackett et al., 1993; Cunningham et al., 1994, mais voir Morrill et al., 2001). À ce titre, la maltraitance est généralement associée à des relations sexuelles non protégées, au partage de seringues et aux rapports sexuels avec des partenaires qui présentent des risques (Cunningham et al., 1994; Hillis et al., 2001; Elliott et al., 2002). En s'appuyant sur des données prospectives, Widom et Kuhns (1996) constatent que l'abus sexuel et la négligence (mais pas l'abus physique) augmentent le risque d'activités de prostitution chez les femmes. Cette même étude précise que les risques de grossesse ou d'activités sexuelles précoces ne sont pas accrus chez les filles maltraitées comparativement aux filles non maltraitées. Quant aux activités sexuelles à risque des garçons, elles ne sont pas associées à la maltraitance dans l'étude de Widom et Kuhns (1996).

Conséquences multiples: Peu d'études ont fait état des conséquences multiples qui découlent des diverses expériences de mauvais traitements durant l'enfance. Les quelques études qui ont été recensées suggèrent que la maltraitance est fortement associée à de multiples conséquences problématiques, à tout le moins durant l'adolescence (Cicchetti et Rogosch, 1997; Egeland, 1997; Trickett et al., 1997; Widom, 1998, 2000a; Thornberry et al., 2001). Encore une fois, la plupart des études qui abordent les effets à long terme reposent sur des mesures rétrospectives (Duncan et al., 1996; Fergusson et Lynskey, 1997; Dube et al., 2002). Force est donc de constater qu'il manque d'études prospectives qui examinent la diversité des expériences de mauvais traitements et leurs multiples conséquences à l'âge adulte, spécifiquement chez les femmes.

Résumé: Considérées dans leur ensemble, les études qui portent sur les mauvais traitements infligés aux filles suggèrent que les conséquences 
de la maltraitance sont passablement nombreuses. Notre recension des écrits indique par contre que les études prospectives sur le sujet sont relativement rares et que celles qui examinent les conséquences de la maltraitance proviennent principalement des travaux effectués par Widom et ses collègues. Bien que les études de Widom aient une valeur certaine, il importe maintenant de tenter d'y répondre en recourant à de nouvelles données.

Par ailleurs, si les études rétrospectives corroborent de façon constante la relation entre les mauvais traitements infligés aux filles et les conséquences négatives à l'adolescence et à l'âge adulte, il importe de souligner certaines réserves méthodologiques. La limite la plus importante concerne le fait de s'appuyer uniquement sur des mesures rétrospectives de la maltraitance (voir Mannarino et al., 1991). De plus, lorsque des études prospectives sont utilisées, la délinquance ou la violence sont souvent les seules conséquences analysées. Ces études attestent que les mauvais traitements en général, et les abus sexuels de l'enfance en particulier, augmentent le risque de délinquance, de violence et d'arrestation. Les études prospectives ont cependant peu étudié les autres conséquences possibles de la maltraitance, à savoir les symptômes dépressifs, l'abus ultérieur d'alcool et de drogues, la violence dans le couple et les comportements sexuels à risque.

Certains chercheurs se sont également attardés aux conséquences négatives de la maltraitance durant l'enfance, à l'adolescence et à l'âge adulte, ou même, au cours de plusieurs cycles de vie. C'est ainsi que Mannarino et al. (1991) se sont intéressés aux conséquences de l'abus sexuel durant l'enfance; Dembo et al. (1990), Banyard et al. (2000), Wolfe et al. (2001) et Herrera et McCloskey (2003), aux conséquences à l'adolescence; Spohn (2000), Widom et Maxfield (2001), Siegel et Williams (2003) et Bergen et al. (2004), aux conséquences à l'âge adulte et Widom et al. (1995), Widom et al. (1999) ainsi que White et Widom (2003), aux conséquences négatives cumulées à travers plusieurs cycles de vie. À la lumière de ces recherches, il est étonnant de constater le peu d'information disponible, issue de données prospectives, sur les conséquences à long terme de la maltraitance des filles. En effet, malgré le fait que le passage de l'adolescence à l'âge adulte soit une période particulièrement importante en ce qui concerne l'adaptation aux rôles d'adulte et aux responsabilités qui en découlent, force est de constater qu'il existe peu de données sur les conséquences de la maltraitance qui s'étendent jusqu'à l'âge adulte. 
Alors qu'une bonne partie de la littérature sur la maltraitance des filles porte sur l'abus sexuel (Kendler et al., 2000; Mannarino et al., 1991 ; Paolucci et al., 2001 ; Putnam, 2003 ; Siegel et Williams, 2003 ; Roberts et al., 2004), d'autres recherches se sont concentrées sur le concept général de la maltraitance (Wolfe et al., 2001). Or, relativement peu de recherches considèrent à la fois l'abus sexuel ainsi que l'abus physique et la négligence dans la définition du concept de maltraitance (Dembo et al., 1990; Widom et al., 1999; Herrera et McCloskey, 2003). Par conséquent, il devient difficile de savoir si l'abus sexuel des filles constitue un facteur de risque unique, plus dommageable que l'abus physique et que la négligence, ou si la maltraitance en général, peu importe la forme qu'elle revêt, compromet le sain développement des filles.

En conclusion, il appert que très peu de recherches ont étudié les conséquences multiples de la maltraitance des filles (ex. : comportements agressifs et violents, comportements intériorisés, problèmes liés à l'alcool et la drogue). De façon générale, la recherche sur les conséquences à long terme de la maltraitance des filles devra: (a) recourir à des études prospectives et longitudinales (b) concevoir des stratégies pour classifier la multiplicité des formes de mauvais traitements incluant l'abus sexuel à l'enfance et (c) prendre en considération les conséquences multiples de la maltraitance (Putnam, 2003). L'étude de Rochester sur le développement des jeunes (Rochester Youth Development Study [RYDS]) sera utilisée afin de répondre aux problèmes soulevés précédemment.

La RYDS est une étude longitudinale toujours en cours et menée auprès d'un échantillon d'adolescents et d'adolescentes choisis au hasard dans la ville de Rochester (New York). Ces adolescents et adolescentes ont participé à la recherche jusqu'à l'âge adulte. Cette étude offre une excellente stratégie de recherche, un échantillon représentatif ainsi qu'une série de mesures qui permettent de produire les connaissances essentielles à la compréhension des conséquences de la maltraitance. À ce jour, les recherches qui ont eu recours aux données de la RYDS ont observé que diverses formes de maltraitance sont liées à des conséquences négatives à l'adolescence (Smith et Thornberry, 1995; Thornberry et al., 2001 ; Ireland et al., 2002) et au début de l'âge adulte (Smith et al., 2004). Cela dit, nos études n'ont pas encore examiné les conséquences de la maltraitance spécifiquement pour les filles. Dans cette étude, nous traiterons des questions de recherche suivantes: 
1. Quelle est la prévalence et la nature des mauvais traitements infligés aux filles durant l'enfance au sein d'un échantillon représentatif?

2. Quelle est la relation, sur le plan bivarié, entre la maltraitance des filles durant l'enfance et l'étendue de ses conséquences à l'âge adulte?

3. Après avoir contrôlé les variables sociodémographiques et familiales et le comportement antisocial antérieur, quelle est la relation entre la maltraitance des filles durant l'enfance et l'étendue de ses conséquences à l'âge adulte?

4. Est-ce que les filles qui ont été abusées sexuellement durant l'enfance sont exposées à plus de conséquences négatives à l'âge adulte, comparativement aux filles non maltraitées et à celles qui ont subi des formes de maltraitance autres que l'abus sexuel?

\section{Méthodologie}

La RYDS a été conçue pour examiner l'évolution de la délinquance et d'autres troubles de comportement dans une population urbaine à haut risque. Le devis de recherche et la stratégie d'échantillonnage ont été décrits en détail dans d'autres publications (Smith et Thornberry, 1995), mais seront rapidement résumés ci-dessous.

\section{Échantillon}

Un échantillon aléatoire stratifié a été constitué à partir d'une population d'élèves des $1^{\text {re }}$ et $2^{\mathrm{e}}$ secondaires des écoles publiques de Rochester en 1987. Afin d'obtenir un nombre suffisant de jeunes à haut risque de délinquance grave et de consommation de drogues, les garçons ont été suréchantillonnés dans un ratio de $3: 1$, ces derniers étant plus susceptibles que les filles de s'engager dans une délinquance sérieuse. De plus, les élèves ont été sélectionnés proportionnellement au taux d'arrestation propre au secteur de recensement de leur résidence. Ces procédures ont permis d'obtenir un échantillon de 1000 élèves (et leurs familles), incluant un nombre total de 271 filles, soit $27 \%$ de l'échantillon global. Bien que l'échantillon final surreprésente les jeunes à haut risque, un continuum de la population adolescente a été intégré à l'échantillon (voir Farnworth et al., 1994). Par ailleurs, puisque la probabilité de sélection de chaque sujet est connue, la stratégie d'échantillonnage permet de pondérer l'ensemble des sujets de manière à ce qu'ils soient représentatifs de la population initiale des élèves des $1^{\text {re }}$ et $2^{\mathrm{e}}$ secondaires choisis dans les écoles publiques de Rochester. Tous les 
résultats non descriptifs qui seront présentés dans cet article ont été pondérés.

Chacun des sujets ainsi que l'autorité responsable de l'enfant - dans la plupart des cas, la mère biologique - ont été interviewés à des intervalles de six mois, du printemps 1988 jusqu'au printemps 1992. Après une interruption de deux ans de la collecte de données, des entrevues annuelles ont débuté en 1994. À la fin de la phase 12 de l'étude, 846 des 1000 sujets initiaux de l'échantillon ont été interviewés de nouveau, ce qui représente un taux global de rétention de $85 \%$. Une comparaison entre les sujets ayant participé jusqu'à la dernière phase et ceux ayant abandonné l'étude en cours de route démontre que les caractéristiques démographiques de ces deux groupes sont similaires (Thornberry et al., 1993). Des informations provenant d'agences officielles ont également été recueillies à partir de différentes sources, notamment le Service de police de Rochester et le Département des services sociaux du comté. Les analyses qui suivent reposent sur les données autorévélées par des adolescentes et leurs parents, sur les arrestations comptabilisées dans les dossiers de la police et sur les situations de mauvais traitements enregistrées par les services sociaux entre la naissance et l'âge de 18 ans approximativement.

\section{Les variables}

Maltraitance: Les données sur les mauvais traitements proviennent des dossiers du Service de protection de l'enfance du Département des services sociaux du comté de Monroe, le comté de résidence des sujets au début de l'étude. Pour chacun des 1000 participants, de leur naissance jusqu'à 1992 alors qu'ils terminaient l'école secondaire, tout incident de maltraitance corroboré par une source a été retenu. Les détails des incidents de maltraitance étaient directement encodés au moyen d'un système de classification élaboré par Cicchetti (Cicchetti et Barnett, 1991). Une description détaillée des procédures de codage est fournie dans l'étude de Barnett et al. (1993) et appliquée aux données de l'étude de Smith et Thornberry (1995) présentées ici. La validité interjuges a été estimée à 0,80 . La prévalence globale des mauvais traitements des filles de notre échantillon est de $27 \%$ (Tableau 1).

Le tableau 1 montre les statistiques descriptives des variables utilisées dans les analyses. La partie gauche du tableau fournit les statistiques 
TABLEA U 1

Statistiques descriptives (seulement pour les femmes, résultats non pondérés)

\begin{tabular}{|c|c|c|c|c|}
\hline & $\begin{array}{c}\% \text { ou } \\
\text { moyenne }\end{array}$ & $\mathrm{N}$ total & $\begin{array}{c}\% \text { ou } \\
\text { moyenne }\end{array}$ & $\mathrm{N}$ listwise \\
\hline \multicolumn{5}{|l|}{ Maltraitance } \\
\hline Absence de maltraitance & 72,7 & $\begin{array}{c}197 \\
(271)\end{array}$ & 75,4 & $\begin{array}{c}150 \\
(199)\end{array}$ \\
\hline Toute forme de maltraitance & 27,3 & $\begin{array}{c}74 \\
(271)\end{array}$ & 24,6 & $\begin{array}{c}49 \\
(199)\end{array}$ \\
\hline \multicolumn{5}{|l|}{ Conséquences à l'âge adulte } \\
\hline Délinquance générale & 48,5 & $\begin{array}{c}115 \\
(237)\end{array}$ & 45,6 & $\begin{array}{c}88 \\
(193)\end{array}$ \\
\hline Délinquance violente & 22,8 & $\begin{array}{c}54 \\
(237)\end{array}$ & 20,7 & $\begin{array}{c}40 \\
(193)\end{array}$ \\
\hline Arrestation & 18,7 & $\begin{array}{c}47 \\
(252)\end{array}$ & 18,8 & $\begin{array}{c}37 \\
(197)\end{array}$ \\
\hline Violence conjugale manifestée & 52,1 & $\begin{array}{c}126 \\
(242)\end{array}$ & 50,5 & $\begin{array}{c}99 \\
(196)\end{array}$ \\
\hline Violence conjugale subie & 33,1 & $\begin{array}{c}80 \\
(242)\end{array}$ & 31,6 & $\begin{array}{c}62 \\
(196)\end{array}$ \\
\hline Consommation de drogues illicites & 38,4 & $\begin{array}{c}91 \\
(237)\end{array}$ & 37,8 & $\begin{array}{c}73 \\
(193)\end{array}$ \\
\hline Consommation problématique d'alcool & 10,3 & $\begin{array}{c}25 \\
(242)\end{array}$ & 10,2 & $\begin{array}{c}20 \\
(196)\end{array}$ \\
\hline Symptômes dépressifs & 26,4 & $\begin{array}{c}64 \\
(242)\end{array}$ & 26,0 & $\begin{array}{c}51 \\
(196)\end{array}$ \\
\hline Risque MTS/HIV au cours de la vie & 47,9 & $\begin{array}{c}116 \\
(242)\end{array}$ & 45,9 & $\begin{array}{c}90 \\
(196)\end{array}$ \\
\hline Conséquences multiples & 2,74 & 242 & 2,65 & 196 \\
\hline \multicolumn{5}{|l|}{ Variables contrôles } \\
\hline Parents ayant complété l'école secondaire & 44,4 & $\begin{array}{c}120 \\
(270)\end{array}$ & 42,2 & $\begin{array}{c}84 \\
(199)\end{array}$ \\
\hline Pauvreté familiale chronique & 35,5 & $\begin{array}{c}77 \\
(217)\end{array}$ & 36,2 & $\begin{array}{c}72 \\
(199)\end{array}$ \\
\hline Changements des figures parentales & 26,8 & $\begin{array}{c}61 \\
(228)\end{array}$ & 25,6 & $\begin{array}{c}51 \\
(199)\end{array}$ \\
\hline \multicolumn{5}{|l|}{ Race/ethnicité } \\
\hline caucasienne & 5,9 & $\begin{array}{c}16 \\
(271)\end{array}$ & 1,0 & $\begin{array}{c}2 \\
(199)\end{array}$ \\
\hline afro-américaine & 79,7 & $\begin{array}{c}216 \\
(271)\end{array}$ & 86,9 & $\begin{array}{c}173 \\
(199)\end{array}$ \\
\hline hispanique & 14,4 & $\begin{array}{c}39 \\
(271)\end{array}$ & 12,1 & $\begin{array}{c}24 \\
(199)\end{array}$ \\
\hline Délinquance à la fin de l'adolescence & 47,3 & $\begin{array}{c}115 \\
(243)\end{array}$ & 45,7 & $\begin{array}{c}91 \\
(199)\end{array}$ \\
\hline
\end{tabular}


descriptives pour l'ensemble des femmes de l'échantillon, alors que la partie droite présente ces statistiques une fois que les valeurs manquantes ont été considérées (listwise). À l'exception de la mesure d'arrestation, tous les indicateurs utilisés au cours de cette étude sont tirés des données rapportées au cours des entrevues des phases 10, 11 et 12, alors que les jeunes femmes étaient au début de la vingtaine.

Délinquance au début de l'âge adulte: La période de référence correspond aux trois années couvertes par les phases 10, 11 et 12. Les entrevues ont été menées à un an d'intervalle et, lors de chacune d'entre elles, les participantes rapportaient les comportements qu'elles avaient adoptés au cours de la dernière année. Les échelles de délinquance autorévélée ont été utilisées dans bon nombre de publications et leur validité est aujourd'hui clairement établie (Thornberry et al., 2003). L'échelle de la délinquance générale comporte des délits allant des infractions mineures, telles que le désordre public et le menu larcin, aux infractions graves, telles que le vol qualifié, l'assaut avec un instrument dangereux et la vente de drogues. Parmi les jeunes femmes de notre échantillon, 48,5\% ont rapporté de la délinquance au début de l'âge adulte (Tableau 1). L'échelle de violence contient six questions concernant la violence dirigée vers autrui, notamment les batailles de gangs, le vol qualifié et les voies de fait. Des gestes de violence ont été rapportés par 22,8\% des participantes. Les données portant sur l'arrestation ont été tirées des dossiers du Service de police de Rochester et des registres de l'État de New York. Étant donné que les dates d'arrestation sont connues, il est possible de savoir lesquelles se sont produites au début de l'âge adulte. Il appert que $18,7 \%$ des femmes de l'échantillon ont à leur actif un rapport d'arrestation au début de leur vingtaine.

La violence dans les relations amoureuses a été évaluée à l'aide du questionnaire sur la résolution des conflits (Conflict Tactics Scale de Straus et Gelles, 1990). Les jeunes femmes qui avaient un conjoint durant au moins une des phases 10 à 12 étaient questionnées sur les situations de conflit avec leur partenaire au cours de cette période, à la fois du point de vue de la violence manifestée et de la violence subie. Pour celles qui n'entretenaient pas de relation intime avec un partenaire au cours de cette période (environ $32 \%$ des répondantes), des résultats nuls leur ont été attribués, indiquant une absence de violence dans les relations amoureuses. L'échelle de violence issue de ce questionnaire comporte neuf questions, dont trois sur la violence mineure et six sur la violence majeure. Au sein de l'échantillon, 52,1\% des jeunes femmes ont rapporté 
avoir posé au moins un acte de violence envers leur partenaire au début de l'âge adulte. Les mêmes questions ont été utilisées pour créer une mesure de la victimisation par le conjoint. Au moins une situation de victimisation a été rapportée par 33,1\% des participantes.

L'échelle de la consommation de drogues contient des questions relatives à la consommation de dix drogues illicites allant de la marijuana à la cocaïne/crack et à l'héroïne. Au total, 38,4\% des femmes ont avoué avoir consommé de la drogue au début de leur vingtaine. Lorsque les répondantes affirmaient avoir consommé de la bière, du vin, des «coolers» ou des boissons fortement alcoolisées, six questions portant sur les comportements problématiques liés à l'alcool (ex. : s'être mis dans le pétrin à l'école, s'être mis dans le pétrin avec la police, s'être bagarré, etc.), étaient posées. Une consommation d'alcool récurrente et donnant lieu à au moins un comportement problématique caractérise $10,3 \%$ des femmes de l'échantillon.

Les symptômes dépressifs font référence à la fréquence de tels symptômes au cours des six derniers mois. Les 14 items de cette échelle, dérivés de la mesure standardisée de Radloff (1977), comprennent des questions telles que «se sentir déprimée ou vraiment triste» ou «être incapable de manger parce que quelque chose te tracasse». Pour chacune des phases 10 à 12 , les résultats ont été dichotomisés à partir $\mathrm{du} 90^{\mathrm{e}}$ percentile. L'obtention d'un résultat supérieur à ce percentile à au moins l'une des phases de l'étude témoigne des tendances dépressives au début de l'âge adulte, ce qui était le cas de 26,4\% des répondantes.

Risque de contracter une MTS ou le VIH: Lors de la phase 11, alors que les participantes étaient âgées d'environ 20 ans, des questions évaluaient si les comportements sexuels des jeunes femmes augmentaient leur risque de développer des maladies transmises sexuellement (MTS) ou le virus d'immunodéficience humaine (VIH) (Survey Research Laboratory, 1987). Les participantes rapportaient si elles avaient contracté une MTS; si elles avaient eu des rapports sexuels en échange d'argent, de drogues ou d'autres avantages; si elles avaient des rapports sexuels avec des partenaires à haut risque (MTS/VIH) et si elles pensaient qu'elles devraient passer un test de dépistage du SIDA. À partir de ces indicateurs, $47,9 \%$ des répondants ont affirmé avoir adopté des comportements sexuels les mettant à risque de contracter une MTS ou le VIH.

Conséquences multiples: Cette variable représente la somme des indicateurs dichotomiques présentés ci-haut à titre de conséquences de la maltraitance. Mentionnons que les infractions violentes ont été exclues 
parce qu'elles avaient déjà été comptabilisées dans le répertoire de la délinquance générale, ce qui aurait occasionné un dédoublement dans les analyses. Cette variable s'étend de 0 à 8 , la moyenne étant de 2,71 conséquences négatives par répondante. Environ $15 \%$ des répondantes n'ont rapporté aucune conséquence négative au début de l'âge adulte, $22 \%$ en ont rapporté une et $63 \%$ en ont rapporté deux ou plus.

Variables contrôles: Cinq variables ont été ajoutées en tant que variables contrôles dans les analyses multivariées. Encore une fois, les indicateurs sont de nature dichotomique, permettant de déterminer les répondantes qui présentent les résultats les plus problématiques. En plus d'être fréquemment utilisés dans les études, de tels indicateurs de risque dichotomisés fournissent une indication claire de la force relative du risque (Farrington et Loeber, 2000).

L'éducation parentale indique si les parents ont terminé l'école secondaire $(44,4 \%)$ ou non $(55,6 \%)$. La pauvreté familiale s'appuie sur des questions issues des 8 premières phases de la collecte de données et qui concernent le chômage ou le recours à l'assistance sociale. Les individus du premier quartile de la distribution cumulative (35,5\%) ont été désignés comme ceux vivant dans une pauvreté chronique. Ils ont reçu de l'assurance-emploi ou de l'assistance sociale pendant six phases ou plus de l'étude. Les transitions familiales informent du nombre de changements de figures parentales au cours de l'adolescence. Le premier quartile de la distribution concerne les participantes qui ont vécu trois transitions et plus $(26,8 \%)$, soit celles qui ont expérimenté un nombre potentiellement problématique de changements en ce qui a trait à la personne chargée de prendre soin d'elles. La race et l'ethnicité incluent les caucasiennes non hispaniques (5,9\%), les afro-américaines $(79,7 \%)$ et les hispaniques $(14,4 \%)$. Mentionnons qu'après avoir tenu compte des valeurs manquantes pour les variables indépendantes, la proportion de femmes caucasiennes dans l'échantillon devient trop petite pour être soumise à des analyses (1,0\% de l'échantillon). Par conséquent, les caucasiennes seront exclues des analyses ultérieures et l'attention sera uniquement dirigée vers les afro-américaines et les hispaniques. La délinquance antérieure réfere à la délinquance générale révélée par les participantes vers la fin de l'adolescence. Cet indicateur est similaire à celui décrit plus haut en référence au début de l'âge adulte. Environ $47 \%$ des femmes ont rapporté avoir été impliquées dans des activités délinquantes à la fin de leur adolescence. 


\section{Résultats}

Le tableau 2 fournit une description détaillée des formes de mauvais traitements ayant été signalés aux services sociaux concernant les jeunes femmes de l'échantillon. Tel que le rapportait le tableau 1, la prévalence de la maltraitance est passablement élevée au sein de l'échantillon, elle se chiffre à $27 \%$. Plus spécifiquement, le tableau 2 met en lumière la distribution des formes de maltraitance subies par les répondantes. Les informations officiellement enregistrées par les services sociaux révèlent

TABLEAU 2

Prévalence des catégories exclusives de maltraitance (afro-américaines et hispaniques, non pondérées)

\begin{tabular}{|c|c|c|c|c|}
\hline & $\%$ & $\mathrm{~N}$ total & $\%$ & $\mathrm{~N}$ listwise \\
\hline \multicolumn{5}{|l|}{ Catégories individuelles de maltraitance } \\
\hline Abus sexuel seulement & 7,58 & 5 & 10,20 & 5 \\
\hline Abus physique seulement & 12,12 & 8 & 6,12 & 3 \\
\hline Négligence seulement & 15,15 & 10 & 16,33 & 8 \\
\hline Abus émotionnel seulement & 9,09 & 6 & 12,24 & 6 \\
\hline Sous-total & 44 & 29 & 45 & 22 \\
\hline \multicolumn{5}{|l|}{ Deux catégories de maltraitance } \\
\hline Abus sexuel et physique & 3,03 & 2 & 2,04 & 1 \\
\hline Abus sexuel et négligence & 1,52 & 1 & 0,00 & 0 \\
\hline Abus sexuel et émotionnel & 3,03 & 2 & 4,08 & 2 \\
\hline Abus physique et négligence & 7,58 & 5 & 8,16 & 4 \\
\hline Abus physique et émotionnel & 13,64 & 9 & 10,20 & 5 \\
\hline Abus émotionnel et négligence & 15,15 & 10 & 18,37 & 9 \\
\hline Sous-total & 44 & 29 & 43 & 21 \\
\hline \multicolumn{5}{|l|}{ Trois catégories du maltraitement } \\
\hline Abus sexuel, physique et émotionnel & 0,00 & 0 & 0,00 & 0 \\
\hline Abus sexuel, émotionnel et négligence & 4,55 & 3 & 2,04 & 1 \\
\hline Abus sexuel, physique et négligence & 1,52 & 1 & 2,04 & 1 \\
\hline Abus physique, émotionnel et négligence & 4,55 & 3 & 6,12 & 3 \\
\hline Sous-total & 11 & 7 & 10 & 5 \\
\hline \multicolumn{5}{|l|}{ Quatre catégories de maltraitance } \\
\hline $\begin{array}{l}\text { Abus sexuel, physique, émotionnel } \\
\text { et négligence }\end{array}$ & 1,52 & 1 & 2,04 & 1 \\
\hline Total des femmes maltraitées & 100 & 66 & 100 & 49 \\
\hline
\end{tabular}


que parmi les jeunes femmes d'origine afro-américaine ou hispanique ayant été maltraitées sexuellement $(\mathrm{n}=15), 33 \%(5 / 15)$ ont subi uniquement cette forme de mauvais traitement alors que pour les autres (67\% ou 10/15), d'autres formes de mauvais traitements s'ajoutaient à l'abus sexuel. Ce chevauchement des formes de maltraitance n'est pas unique aux victimes d'abus sexuel. Il peut également être observé chez les filles qui ont été abusées physiquement ou émotivement et chez celles qui ont été négligées. Il importe de souligner que cette évaluation de la prévalence des mauvais traitements au sein de notre échantillon sous-estime les situations réelles de maltraitance, puisque seulement les incidents qui ont été rapportés aux services sociaux et dont on a pu fournir la preuve ont été retenus.

TABLEAU 3

Relation bivariée entre la maltraitance officielle et ses conséquences au début de l'âge adulte (afro-américaines et hispaniques seulement)

\begin{tabular}{|lcc|}
\hline & \multicolumn{1}{c|}{$\begin{array}{c}\text { Pas de } \\
\text { maltraitance (\%) }\end{array}$} & $\begin{array}{c}\text { Toute forme } \\
\text { de maltraitance (\%) }\end{array}$ \\
\hline $\begin{array}{l}\text { Délinquance générale * } \\
(\mathrm{N}=211)\end{array}$ & 44,8 & 59.9 \\
\hline $\begin{array}{l}\text { Délinquance violente ** } \\
(\mathrm{N}=211)\end{array}$ & 21,6 & 36,3 \\
\hline $\begin{array}{l}\text { Arrestation** } \\
(\mathrm{N}=223)\end{array}$ & 11,9 & 31,8 \\
$\begin{array}{l}\text { Violence conjugale manifestée ** } \\
(\mathrm{N}=215)\end{array}$ & 41,7 & 63,2 \\
$\begin{array}{l}\text { Violence conjugale subie ** } \\
(\mathrm{N}=215)\end{array}$ & 24,7 & 42,7 \\
\hline $\begin{array}{l}\text { Consommation de drogues illicites } \\
(\mathrm{N}=211)\end{array}$ & 33,4 & 44,5 \\
\hline $\begin{array}{l}\text { Consommation problématique d'alcool ** } \\
(\mathrm{N}=215)\end{array}$ & 8,2 & 18,1 \\
\hline $\begin{array}{l}\text { Symptômes dépressifs } \\
(\mathrm{N}=215)\end{array}$ & 22,4 & 30,2 \\
\hline $\begin{array}{l}\text { Risque MTS/VIH au cours de la vie ** } \\
(\mathrm{N}=215)\end{array}$ & 42,0 & 62,6 \\
\hline
\end{tabular}

${ }^{*} \mathrm{p}<0,10$ (two-tailed); ${ }^{*} \mathrm{p}<0,05$ (two-tailed)

Note: Nous avons effectué des analyses bivariées supplémentaires sur l'échantillon des répondantes inclus dans les analyses multivariées du tableau 4. Ces résultats peuvent être obtenus en contactant le premier auteur. La fluctuation de certaines relations est attribuable aux valeurs manquantes et à la réduction de la taille de l'échantillon. Dans le cas présent, nous avons décidé d'utiliser tous les cas disponibles afin de maximiser la taille de l'échantillon et le nombre de femmes maltraitées inclus dans les analyses. 
T A B LEA U 4

Les conséquences de la maltraitance au début de l'âge adulte chez les afro-américaines et les hispaniques (les rapports de cote sont issus de régressions logistiques)

\begin{tabular}{|c|c|c|c|c|c|c|c|c|c|c|}
\hline & $\begin{array}{l}\text { Délinquance } \\
\text { générale } \\
(\mathrm{N}=179)\end{array}$ & $\begin{array}{l}\text { Délinquance } \\
\text { violente } \\
(\mathrm{N}=179)\end{array}$ & $\begin{array}{c}\text { Arrestation } \\
(\mathrm{N}=181)\end{array}$ & $\begin{array}{c}\text { Violence } \\
\text { conjugale } \\
\text { manifestée } \\
(\mathrm{N}=180)\end{array}$ & $\begin{array}{c}\text { Violence } \\
\text { conjugale } \\
\text { subie } \\
(\mathrm{N}=180)\end{array}$ & $\begin{array}{l}\text { Consomma- } \\
\text { tion de } \\
\text { drogues } \\
\text { illicites } \\
(\mathrm{N}=179)\end{array}$ & $\begin{array}{l}\text { Problèmes } \\
\text { liés à } \\
\text { l'alcool } \\
(\mathrm{N}=180)\end{array}$ & $\begin{array}{c}\text { Symptômes } \\
\text { dépressifs } \\
(\mathrm{N}=180)\end{array}$ & $\begin{array}{l}\text { Risque } \\
\text { MTS/HIV } \\
(\mathrm{N}=180)\end{array}$ & $\begin{array}{l}\text { Conséquences } \\
\text { multiples } s^{\mathrm{a}} \\
(\mathrm{N}=180)\end{array}$ \\
\hline Toute forme de maltraitance & 1,28 & 1,56 & $2,33^{*}$ & $3,23^{* *}$ & 1,87 & $1,98^{*}$ & 1,30 & 1,04 & 1,15 & $0,79 * *$ \\
\hline Parents ont complété leur secondaire & $2,69 * *$ & 1,48 & $0,13^{* *}$ & 1,18 & 1,47 & 0,73 & 0,82 & $0,47^{*}$ & 0,59 & $-0,16$ \\
\hline $\begin{array}{l}\text { Changements multiples } \mathrm{p} / \mathrm{r} \\
\text { aux figures parentales }\end{array}$ & 1,06 & $0,42^{*}$ & 1,40 & 1,04 & 1,22 & 0,97 & 2,00 & 0,89 & 1,20 & 0,15 \\
\hline Pauvreté chronique & 1,20 & 1,51 & 1,13 & 0,69 & 1,35 & 1,19 & 2,01 & 1,27 & 0,94 & 0,18 \\
\hline $\begin{array}{l}\text { Délinquance à la fin de } \\
\text { l'adolescence }\end{array}$ & 2,67 ** & $2,88^{* *}$ & $5,68^{* *}$ & $1,98 * *$ & 2,51 ** & $3,06^{* *}$ & 13,93 ** & $2,42^{* *}$ & 1,47 & $1,46^{* *}$ \\
\hline Pseudo- $\mathrm{R}^{2}$ de Nagelkerke & 13,9 & 9,1 & 30,3 & 12,6 & 10,3 & 13,1 & 28,7 & 9,1 & 12,4 & $14,68 * *$ \\
\hline
\end{tabular}

* $\mathrm{p}<0,10$ (two-tailed); ** $\mathrm{p}<0,05$ (two-tailed)

${ }^{a}$ Pour l'équation des conséquences multiples, la régression de type OSL a été utilisée au lieu d'une régression logistique. Les coefficients présentés sont des coefficients de régression et la statistique résumée est le $\mathrm{R}^{2}$ ajusté. 
De surcroît, alors que $44 \%$ des filles qui ont subi des mauvais traitements n'ont été exposées qu'à une seule forme de maltraitance, 56\% ont subi des formes multiples de maltraitance à leur actif. Au total, 12\% des filles maltraitées comptaient au moins trois formes de mauvais traitements dans les dossiers des services sociaux. Afin de tenir compte de ce chevauchement significatif des types de maltraitance, nous avons eu recours à une mesure globale de toutes les formes de mauvais traitements pour les analyses présentées aux tableaux 3 et 4 .

Les résultats des analyses bivariées montrant le lien entre les mauvais traitements enregistrés par les services sociaux durant l'enfance ou à l'adolescence et leurs conséquences négatives au début de l'âge adulte sont présentés au tableau 3. Pour chacune des conséquences, un test d'indépendance du khi-carré indique si des différences significatives s'établissent entre les filles non maltraitées et celles qui l'ont été sous une forme ou une autre. À l'exception de la consommation de drogues illicites et des symptômes dépressifs, les résultats des analyses bivariées indiquent que le fait d'avoir été victime de mauvais traitements a des répercussions sur le développement des femmes à l'aube de l'âge adulte.

Le tableau 4 présente les résultats des analyses multivariées issues de dix équations (9 régressions logistiques et une régression linéaire). Les conséquences de la maltraitance figurent sur la première ligne (à titre de variables dépendantes). Pour chacune de ces conséquences, le risque relatif à la maltraitance est indiqué sous forme de rapport de cote (odds ratio) tout en contrôlant statistiquement d'autres variables. Pour chacune des équations, le modèle statistique du khi-carré, qui est de manière générale analogue au test $\mathrm{F}$ d'une régression linéaire, indique si des coefficients sont statistiquement significatifs. Les résultats révèlent que pour chacune des équations estimées, le modèle statistique de khi-carré est significatif. Après avoir contrôlé les variables sociodémographiques, les caractéristiques familiales et l'implication dans la délinquance à l'adolescence, plusieurs des relations qui étaient significatives dans les analyses bivariées ne le sont plus dans les analyses multivariées. Néanmoins, tous les rapports de cote de la maltraitance vont dans la direction prédite: lorsque des incidents de maltraitance sont signalés, plus grands sont les risques de subir des conséquences négatives au début de l'âge adulte.

Plus concrètement, le fait d'avoir subi de mauvais traitements augmente les risques d'être arrêté, d'employer de la violence contre son conjoint et de consommer des drogues. Le cumul des conséquences 
négatives est aussi plus probable ${ }^{2}$. Toutefois, il importe de mentionner que l'expérience de la maltraitance chez les femmes de notre échantillon n'augmente pas le risque de s'adonner à une délinquance générale ou à des infractions violentes, d'être victime de violence conjugale, d'avoir des problèmes liés à l'alcool, de manifester des symptômes dépressifs ou d'adopter des comportements sexuels à risque en regard des MTS et du VIH ${ }^{3}$.

Finalement, d'autres analyses bivariées ont été effectuées (tableau 5). Considérant qu'une grande partie des recherches sur la maltraitance des filles s'est exclusivement concentrée sur l'abus sexuel, la variable de la maltraitance a été subdivisée en trois catégories: pas de mauvais traitements, les mauvais traitements qui n'incluent pas d'abus sexuel et les abus sexuels. L'objectif était de tester différents modèles multivariés. Or, puisque le nombre de filles de notre échantillon pour qui des abus sexuels ont été enregistrés est mince $(n=15)$ et puisque ce nombre baisse à 11 en tenant compte des valeurs manquantes des variables indépendantes (tableau 2), il est impossible de procéder à des analyses multivariées. Par conséquent, le tableau 5 présente les résultats des ana-

2. La taille de l'échantillon varie entre les tableaux 3 et 5 , comparativement au tableau 4. En effet, pour les modèles bivariés (tableaux 3 et 5), le nombre de répondants s'étend d'un minimum de 211 à un maximum de 223 (pondéré). Or, pour les modèles multivariés, compte tenu des valeurs manquantes pour les variables contrôles, la taille de l'échantillon s'étend de 179 à 181 répondants (pondéré). Certaines des variations observées entre les résultats des modèles bivariés et multivariés peuvent être attribuées aux valeurs manquantes. Plus précisément, certains examinateurs ont pointé du doigt l'absence de relation significative entre la maltraitance et l'usage de drogues illicites à un niveau d'analyse bivariée, alors que cette même relation était significative lors des analyses multivariées. Une interprétation possible de ce genre de résultats concerne la présence d'une suppression statistique. Néanmoins, sans une exploration plus poussée des données, l'interprétation des résultats concernant l'usage de drogues illicites devra se concentrer sur les valeurs manquantes. Par conséquent, la relation qui existe entre la maltraitance et l'usage de drogues illicites devra être traitée avec prudence.

3. Dans des analyses qui ne sont pas présentées ici, nous avons testé un modèle où les variables suivantes ont été intégrées: la présence d'une délinquance générale vers la fin de l'adolescence (variable dépendante), les diverses formes de mauvais traitements (variable indépendante), ainsi que les variables sociodémographiques et les caractéristiques familiales (variables contrôles). Les mauvais traitements ne sont pas significativement liés à la délinquance générale vers la fin de l'adolescence chez les femmes de l'étude de Rochester. Par conséquent, la délinquance à la fin de l'adolescence n'agit pas comme un modérateur entre la maltraitance et les conséquences de la maltraitance au début de l'âge adulte. Par la suite, chacune des équations du tableau 4 ont été réévaluées en ne tenant pas compte de l'effet de la délinquance à l'adolescence. Les conséquences négatives de la maltraitance au début de l'âge adulte rapportées dans le tableau 4 sont pour la plupart demeurées stables. 
TABLEAU 5

Relations bivariées entre l'abus sexuel, les autres formes de maltraitance et les conséquences au début de l'âge adulte (afro-américaines et hispaniques seulement)

Cas de mauvais traitements enregistrés par les instances officielles

\begin{tabular}{|c|c|c|c|}
\hline & $\begin{array}{l}\text { Aucun abus } \\
(\%)\end{array}$ & $\begin{array}{c}\text { Abus physique/ } \\
\text { négligence/ } \\
\text { abus émotionnel (\%) }\end{array}$ & $\begin{array}{c}\text { Abus } \\
\text { sexuel } \\
(\%)\end{array}$ \\
\hline $\begin{array}{l}\text { Délinquance générale ** } \\
(\mathrm{N}=211)\end{array}$ & 44,8 & 54,8 & 87,9 \\
\hline $\begin{array}{l}\text { Délinquance violente * } \\
(\mathrm{N}=211)\end{array}$ & 21,6 & 36,6 & 34,7 \\
\hline $\begin{array}{l}\text { Arrestation ** } \\
(\mathrm{N}=223)\end{array}$ & 11,9 & 33,8 & 22,6 \\
\hline $\begin{array}{l}\text { Violence conjugale manifestée } * * \\
(\mathrm{~N}=215)\end{array}$ & 41,7 & 57,6 & 94,6 \\
\hline $\begin{array}{l}\text { Violence conjugale subie ** } \\
(\mathrm{N}=215)\end{array}$ & 24,7 & 36,1 & 79,1 \\
\hline $\begin{array}{l}\text { Consommation de drogues illicites** } \\
(\mathrm{N}=211)\end{array}$ & 33,4 & 38,6 & 76,5 \\
\hline $\begin{array}{l}\text { Consommation d'alcool problématique * } \\
(\mathrm{N}=215)\end{array}$ & 8,2 & 16,2 & 28,8 \\
\hline $\begin{array}{l}\text { Symptômes dépressifs } \\
(\mathrm{N}=215)\end{array}$ & 22,4 & 27,9 & 43,1 \\
\hline $\begin{array}{l}\text { Risque MTS/VIH au cours de la vie }{ }^{* *} \\
(\mathrm{~N}=215)\end{array}$ & 42,0 & 59,0 & 82,5 \\
\hline
\end{tabular}

${ }^{*} \mathrm{p}<0,10$ (two-tailed); ${ }^{* *} \mathrm{p}<0,05$ (two-tailed)

Note: Voir la note du tableau 3. Les analyses bivariées effectuées avec l'échantillon des sujets inclus dans les analyses multivariées du tableau 4 indiquent un pattern général de résultats très similaire. Les résultats peuvent être obtenus en contactant le premier auteur.

lyses bivariées, lesquels devront nécessairement être interprétés avec précaution.

Les résultats du tableau 5 suggèrent que les filles ayant été maltraitées sexuellement, que ce soit en association ou non avec d'autres formes de mauvais traitements, semblent plus à risque d'en subir des conséquences développementales négatives comparativement à leurs consœurs ayant été maltraitées sans toutefois avoir été abusées sexuellement. À l'exception des arrestations et des infractions violentes, il appert que les filles qui ont été abusées sexuellement sont plus à risque, au début de l'âge adulte, de commettre des actes de délinquance générale, de 
poser des gestes de violence envers leur conjoint, de subir de la violence conjugale, de consommer des drogues illicites, d'avoir des problèmes de consommation d'alcool et d'adopter des comportements sexuels à risque de MTS/HIV. Dans certains cas, l'écart qui sépare les femmes qui ont subi de mauvais traitements sans être abusées sexuellement de celles qui ont été abusées sexuellement est particulièrement marqué. À titre d'exemple, il semble que l'abus sexuel, en comparaison des autres formes de maltraitance, augmente le risque d'adopter des comportements violents envers le conjoint de $64 \%$, le risque de consommer des drogues illicites de $98 \%$ et le risque de contracter des MTS ou le VIH de $40 \%$. Si les résultats présentés au tableau 5 sont surprenants, il reste difficile d'établir un constat clair et définitif sur les conséquences de l'abus sexuel des filles à partir de nos analyses bivariées; des analyses multivariées seront nécessaires avant d'établir des conclusions plus fermes.

\section{Conclusion}

Nous avons exploré les conséquences à long terme de la maltraitance officielle chez les filles en utilisant les données de l'étude de Rochester sur le développement des jeunes (RYDS). En s'appuyant sur les recherches antérieures dans ce domaine, nous avons examiné lors de nos premières analyses la distribution des expériences de mauvais traitements au sein de notre échantillon. Les données de la RYDS indiquent que la majorité des filles maltraitées ont subi des formes multiples de mauvais traitements. Par conséquent, nos analyses se sont d'abord appuyées sur une mesure globale des expériences de mauvais traitements.

Ensuite, nous avons estimé une série de tables de contingence où la relation entre la maltraitance et ses conséquences négatives à l'âge adulte a été analysée à partir de divers indicateurs. Les résultats bivariés indiquent que la maltraitance, toutes formes confondues, durant l'enfance ou à l'adolescence chez les filles de la RYDS est associée à plusieurs conséquences négatives lors du passage à l'âge adulte. Toutefois, les hypothèses concernant la relation positive entre la maltraitance et la consommation de drogues illicites, ainsi qu'entre la maltraitance et les symptômes dépressifs ont été infirmées.

Par ailleurs, les analyses multivariées offrent des résultats intéressants. En effet, il appert que les filles qui ont subi de mauvais traitements dont 
on a pu fournir la preuve ont plus de chances de se faire arrêter au début de l'âge adulte. Un tel résultat concorde avec ceux d'autres études prospectives ayant utilisé des données officielles comme variable dépendante (Spohn, 2000; Widom et Maxfield, 2001; Siegel et Williams, 2003). Néanmoins, lorsque la délinquance est estimée au moyen de données autorévélées plutôt qu'avec des sources officielles, son lien avec la maltraitance devient non significatif. Plus précisément, alors que les liens entre la maltraitance et la délinquance générale, ainsi qu'entre la maltraitance et la violence, étaient constatés dans les analyses bivariées, ces liens disparaissent complètement dans les analyses multivariées. Or, si plusieurs études prospectives ont établi une relation entre la maltraitance des filles et l'ampleur de leur délinquance autorévélée à l'adolescence (Herrera et McCloskey, 2003; Swanston et al., 2003), aucune ne semble avoir considéré la délinquance générale et la violence autorévélées au début de l'âge adulte.

À l'instar des résultats de White et Widom (2003), nous avons pu observer que les filles maltraitées rapportent plus souvent user de violence dans le cadre d'une relation amoureuse que celles qui n'ont aucune histoire de maltraitance. Par contre, les résultats indiquent que la maltraitance n'augmente pas le risque d'être victime de violence conjugale. Par ailleurs, conformément aux résultats de l'étude prospective de Horwitz et al. (2001), il ne semble pas exister de relation entre la maltraitance des filles et la présence de symptômes dépressifs au début de l'âge adulte. Malgré tout, il importe de souligner que l'absence d'une telle relation défie une grande partie des recherches transversales rétrospectives qui, elles, ont démontré l'existence d'une relation entre ces deux dimensions (Kendler et al., 2000).

La relation significative rapportée dans les analyses multivariées entre les mauvais traitements durant l'enfance et la consommation de drogues à l'âge adulte est surprenante considérant l'absence de résultats significatifs entre ces deux variables lors des analyses bivariées. À ce titre, l'étude prospective de Ireland et Widom (1994) a démontré la présence d'un risque plus élevé d'arrestation pour des délits liés à l'alcool et aux drogues chez les femmes ayant subi de la maltraitance, alors que Widom et al. (1999), en s'appuyant sur des données autorévélées, n'observent pas de relation significative entre la maltraitance et l'abus de drogues. Cette absence de relation entre les expériences de maltraitance des femmes et leurs problèmes d'alcool au début de l'âge adulte défie les recherches antérieures sur le sujet (Widom et al., 1995). Mentionnons toutefois que 
Widom et al. (1995) avaient alors utilisé des mesures de la prévalence à vie de problèmes de consommation d'alcool, alors que notre étude se limitait au début de l'âge adulte. Enfin, considérant la rareté des études prospectives portant sur la relation entre la maltraitance et les comportements sexuels à risque ultérieurs, nos résultats constituent une des premières pierres d'assise dans ce domaine et plus particulièrement en ce qui concerne les risques de contracter une MTS ou le VIH. Finalement, nos résultats indiquent que les mauvais traitements enregistrés par les agences officielles augmentent le risque de subir des conséquences multiples chez les femmes au début de l'âge adulte.

Bien qu'ils s'inscrivent dans une optique exploratoire, les résultats propres à l'abus sexuel démontrent la nécessité de considérer les conséquences particulières de cette forme de maltraitance lors du passage des filles à l'âge adulte. Les résultats bivariés indiquent les mauvais traitements, toutes formes confondues, qui risquent de compromettre le développement des femmes à l'aube de l'âge adulte. Le risque que représentent les mauvais traitements semble s'amplifier lorsque les filles sont victimes d'abus sexuel.

\section{Discussion}

Considérant que la maltraitance des filles est associée à plusieurs conséquences négatives particulières au début de l'âge adulte, ainsi qu'au cumul de ces conséquences, il serait fort étonnant qu'une seule trajectoire développementale puisse expliquer les conséquences de la maltraitance chez les jeunes femmes.

Par ailleurs, bien que nos analyses multivariées indiquent que la maltraitance ne provoque pas les comportements violents autorévélés à l'âge adulte, ce qui laisserait croire à un cycle de la violence, un tel cycle semble toutefois s'opérer lorsque la violence est exercée à l'endroit du conjoint. Les femmes qui ont été maltraitées à un jeune âge sont plus enclines que celles n'ayant pas subi de mauvais traitements à manifester de la violence à l'endroit de leur conjoint. Une des implications possibles de ce résultat concerne la transmission intergénérationnelle du cycle de la violence. Une grande proportion des jeunes femmes de notre échantillon sont devenues mères alors qu'elles étaient encore adolescentes $(40 \%)$ et plusieurs autres étaient mères au début de la vingtaine. La diversité des liens possibles entre la maltraitance et les conséquences 
négatives, telles que la violence conjugale, la consommation abusive de drogues et les risques d'arrestation, suggère qu'il existe des risques significatifs pour les enfants de la prochaine génération. La consommation de drogues par les parents constitue d'ailleurs un des problèmes majeurs des enfants desservis par les services de protection de l'enfance (Kang et al., 1999). Plus encore, la persistance du comportement antisocial et les relations agressives avec autrui peuvent mener à la maltraitance des enfants et à l'émergence d'un comportement antisocial chez la prochaine génération (Pears et Capaldi, 2001).

Des études additionnelles seront nécessaires avant de pouvoir affirmer avec plus de certitude que les formes de maltraitance qui impliquent l'abus sexuel ont des conséquences particulièrement sérieuses. Bien que nous ne soyons pas en mesure d'estimer des modèles multivariés ou d'évaluer adéquatement les mécanismes en cause, certains suggèrent que les nouvelles recherches sur les effets neurobiologiques du traumatisme et du stress extrême sont prometteuses (Heim et al., 2000; Roberts et al., 2004). Un des plus récents postulats de la recherche est que le symptôme de stress post-traumatique partage des caractéristiques avec d'autres troubles anxieux, ce qui aurait comme conséquence d'activer une série de réactions physiologiques telles que l'hypervigilance et l'anxiété menant à de nombreux problèmes d'adaptation à long terme.

Par ailleurs, il importe de souligner les limites de cette étude. Le nombre relativement faible de filles de notre échantillon pour lesquelles de mauvais traitements ont été signalés a contribué à affaiblir notre capacité à détecter les effets de la maltraitance. Une des limites connexes est que notre échantillon est trop petit pour distinguer les stades de développement au cours desquels les conséquences de la maltraitance surgissent. Nos recherches précédentes, qui incluaient les garçons, avaient permis de démontrer que la maltraitance à l'adolescence et la maltraitance récurrente avaient des effets à plus long terme que la maltraitance limitée à l'enfance (Thornberry et al., 2001; Ireland et al., 2002). Une autre limite repose sur le recours à une source officielle de la maltraitance pour établir la présence ou l'absence de mauvais traitements dans notre échantillon. Comme l'ont souligné d'autres chercheurs, les cas de mauvais traitements rapportés par les répondantes et ceux qui sont enregistrés par les agences officielles ne coïncident pas toujours (Widom et Shepard, 1996). Finalement, l'absence des filles de race blanche au sein de notre étude, due à un nombre trop élevé de valeurs 
manquantes, limite la possibilité de généraliser les résultats à l'ensemble de la population.

En terminant, bien que nous nous soyons principalement attardés aux conséquences à long terme des mauvais traitements chez les filles, il demeure que la capacité de résilience par rapport aux mauvais traitements ressort des données de l'étude de Rochester. L'absence de conséquences problématiques chez un certain nombre d'enfants maltraités constitue un fait largement accepté dans les recherches prospectives, et ce, particulièrement quand les mauvais traitements se rattachent à un seul incident ou à un incident ayant eu lieu au cours de la petite enfance (McCloskey, 1997 ; Ireland et al., 2002; Edwards et al., 2004). Il existe plusieurs explications possibles à la résilience, notamment l'influence positive d'une intervention ou du processus de maturation de l'enfant (Widom, 2000b). Par exemple, il est possible que les conséquences des mauvais traitements s'estompent avec le temps si ces incidents ne sont pas répétés. Les enfants et leurs familles sont remplis de ressources et capables de résister à des événements difficiles. Aussi, il est possible de croire qu'après une série d'événements stressants, ils développent la capacité de se remettre sur pied. Nul ne saurait donc insister suffisamment sur la nécessité de bien comprendre les processus par lesquels passe la résilience afin de développer des stratégies d'intervention efficaces.

\section{Références}

Arnett, J.J. (2000). Emerging adulthood: a theory of development from the late teens through the twenties. American Psychologist, 55, 469-480.

Bagley, C., \& Mallick, K. (2000). Prediction of sexual, emotional and physical maltreatment and mental health outcomes in a longitudinal cohort of 290 adolescent women. Child Maltreatment, 5, 218-226.

Banyard, V.L., Arnold, S., \& Smith, J. (2000). Childhood sexual abuse and dating experiences of undergraduate women. Child Maltreatment, 5, 39-48.

Barnett, D., Manly, J.T., \& Cicchetti, D. (1993). Defining child maltreatment: the interface between policy and research. In D. Cicchetti \& S.L. Toth (eds), Child abuse, child development, and social policy. Norwood, NJ: Ablex.

Bergen, H.A., Martin, G., Richardson, A.S., Allison, S., \& Roeger, L. (2004). Sexual abuse, antisocial behaviour and substance use: gender differences in young community adolescents. Australian and New Zealand journal of psychiatry, 38, 34-41.

Bolger, K.E., \& Patterson, C.J. (2001). Developmental pathways from child maltreatment to peer rejections. Child Development, 72, 549-568. 
Briere, J. (1992). Methodological issues in the study of sexual abuse effects. Journal of Consulting and Clinical Psychology, 60, 196-203.

Brown, J., Cohen, P., Johnson, J.G., \& Smailes, E.M. (1999). Childhood abuse and neglect: specificity of effects on adolescent and young adult depression and suicidality. Journal of the American academy of child and adolescent psychiatry, 38, 1490-1496.

Bureau of Justice Statistics. (1994). Special report: Women in prison. Washington, DC: U.S. Department of Justice.

Buzi, R.S., Tortolero, S.R., Roberts, R.E., Ross, M.W., Addy, R.C., \& Markham, C.M. (2003). The impact of a history of sexual abuse on high-risk sexual behaviors among females attending alternative schools. Adolescence, 38, 595605.

CAPTA, 42 U.S.C.A. 5106g. Federal child abuse, prevention, and treatment act.

Chandy, J.M., Blum, R.W., \& Resnick, M.D. (1996). Gender-specific outcomes for sexually abused adolescents. Child abuse and neglect, 20, 1219-1231.

Chen, X., Tyler, K.A., Whitbeck, L.B., \& Hoyt, D.R. (2004). Early sexual abuse, street adversity, and drug use among female homeless and runaway adolescents in the Midwest. Journal of drug issues, 34, 1-22.

Chesney-Lind, M., \& Shelden, R.G. (1998). Girls, delinquency and juvenile justice (2nd ed.). Belmont, CA : Wadsworth.

Cicchetti, D., \& Barnett, D. (1991). Toward the development of a scientific nosology of child maltreatment. In D. Cicchetti \& W.M. Grove (eds), Thinking clearly about psychology: essays in honor of Paul E. Meehl, vol. 1 : Matters of public interest; vol. 2: Personality and psychopathology. Minneapolis, MN: University of Minnesota Press.

Cicchetti, D., Rogosch, F.A., Lynch, M., \& Holt, K.D. (1993). Resilience in maltreated children: processes leading to adaptive outcomes. Development and Psychopathology, 5, 629-647.

Cicchetti, D., \& Rogosch, F.A. (1997). The role of self-organization in the promotion of resilience in maltreated children. Development and Psychopathology, 9, 797-815.

Cicchetti, D., \& Toth, S. (1997). Developmental perspectives on trauma: theory, research, and intervention: Rochester symposium on developmental psychology, vol. 8 . Rochester, NY: University of Rochester Press.

Crick, N.R., \& Dodge, K.A. (1994). A review and reformulation of social information-processing mechanisms in children's social adjustment. Psychological Bulletin, 115, 74-101.

Crittenden, P.M., Claussen, A.H., \& Sugarman, D.B. (1994). Physical and psychological maltreatment in middle childhood and adolescence. Development and Psychopathology, 6, 145-164.

Cunningham, R.M., Stiffman, A.R., Dore, P., \& Earls, F. (1994). The association of physical and sexual abuse with HIV risk behaviors in adolescence and young adulthood: implications for public health. Child abuse and neglect, 18, 233-245.

Daly, K. (1994). Gender, crime and punishment. New Haven, CT : Yale University Press. 
Dembo, R., Getreu, A., Williams, L., Berry, E., La Voie, L., Genung, L., Schmeidler, J., Wish, E.D., \& Kern. J. (1990). A longitudinal study of the relationships among alcohol use, marijuana/hashish use, cocaine use, et emotional/psychological functioning problems in a cohort of high-risk youths. The International Journal of the Addictions, 25, 1341-1382.

Dodge, K.A., Pettit, G.S., \& Bates, J.E. (1997). How the experience of early physical abuse leads children to become chronically aggressive. In D. Cicchetti et S.L. Toth (eds), Developmental perspectives on trauma: theory, research, and intervention: Rochester symposium on developmental psychology, vol. 8. Rochester, NY: University of Rochester Press.

Dube, S.R., Felitti, V.J., Dong, M., Chapman, D.P., Giles, W.H., \& Anda, R.F. (2002). Childhood abuse, neglect, and household dysfunction and the risk of illicit drug use: the adverse childhood experiences study. Pediatrics, 111, 564-572.

Duncan, R., Saunders, B., Kilpatrick, D., Hanson, R., \& Resnick, H. (1996). Childhood physical assault as a risk factor for PTSD, depression, et substance abuse: findings from a national survey. American Journal of Orthopsychiatry, 66, 437-448.

Dunlap, E., Golub, A., \& Johnson, B.D. (2003). Girls' sexual development in the inner city: from compelled childhood sexual contact to sex-for-things exchanges. Journal of Child Sexual Abuse, 12, 73-96.

Edwards, V.J., Holden, G.W., Felitti, V.J., \& Anda, R.F. (2003). Relationship between multiple forms of childhood maltreatment and adult mental health in community respondents: results from the adverse childhood experiences study. The American Journal of Psychiatry, 160, 1453-1460.

Edwards, V.J., Anda, R.F., Felitti, V.J., \& Dube, S.R. (2004). Adverse childhood experiences and health-related quality of life as an adult. In K.A KendallTackett (ed.), Health consequences of abuse in the family: a clinical guide for evidencebased practice. Application and practice in bealth psychology. Washington, DC: American Psychological Association.

Egeland, B. (1997). Mediators of the effects of child maltreatment on developmental adaptation in adolescence. In D. Cicchetti \& S.L. Toth (eds), Developmental perspectives on trauma: theory, research, and intervention: Rochester symposium on developmental psychopathology, vol. 8. Rochester, NY: University of Rochester Press.

Elliott, G.C., Avery, R., Fishman, E., \& Hoshiko, B. (2002). The encounter with family violence and risk sexual activity among young adolescent females. Violence and Victims, 17, 569-592.

Farnworth, M., Thornberry, T.P., Krohn, M.D., \& Lizotte, A.J. (1994). Measurement in the study of class and delinquency: integrating theory and research. Journal of research in crime and delinquency, 31, 32-61.

Farrington, D.P., \& Loeber, R. (2000). Some benefits of dichotomization in psychiatric and criminological research. Criminal Behaviour and Mental Health, 10, 100-122.

Fergusson, D.M., \& Lynskey, M.T. (1997). Physical punishment/maltreatment during childhood and adjustment in young adulthood. Child abuse and neglect, 21, 617-630. 
Follingstad, D.R., Wright, S., Lloyd, S., \& Sebastian, J.A. (1991). Sex differences in motivations and effects in dating violence. Family Relations, 40, 51-57.

Foo, L., \& Margolin, G. (1995). A multivariate investigation of dating aggression. Journal of Family Violence, 10, 351-377.

Furstenberg, F.F. (1990). Coming of age in a changing family system. In S. Feldman \& G. Elliott (eds), At the threshold: the developing adolescent (147170). Cambridge, MA: Harvard University Press.

Graham-Bermann, S.A., \& Levendosky, A.A. (1998). Traumatic stress symptoms in children of battered women. Journal of Interpersonal Violence, 14, 11-128.

Gutierres, S.E., \& Todd, M. (1997). The impact of childhood abuse on treatment outcomes of substance users. Professional psychology: Research and practice, 28, 348-354.

Heim, C., Newport, D.J., Heit, S., Graham, Y.P., Wilcox, M., Miller, A.H., \& Nemeroff, C.B. (2000). Pituitary-adrenal and autonomic responses to stress in women after sexual and physical abuse in childhood. Journal of the American Medical Association, 284, 592-597.

Herrera, V. \& McCloskey, L.A. (2001). Gender differences in the risk for delinquency among youth exposed to family violence. Child abuse and neglect, 25, 1037-1051.

Herrera, V., \& McCloskey, L.A. (2003). Sexual abuse, family violence, and female delinquency : findings from a longitudinal study. Violence and Victims, 18, 319-334.

Hillis, S.D., Anda, R.F., Felitti, V.J., \& Marchbanks, P.A. (2001). Adverse childhood experiences and sexual risk behaviors in women: a retrospective cohort study. Family planning perspectives, 33, 206-211.

Hollister-Wagner, G.H., Foshee, V.A., \& Jackson, C. (2001). Adolescent aggression: models of resiliency. Journal of Applied Social Psychology, 31, 445-466.

Horwitz, A.V., Widom, C.S., McLaughlin, J., \& White, H.R. (2001). The impact of childhood abuse and neglect on adult mental health : a prospective study. Journal of Health and Social Behavior, 42, 184-201.

Hoyt, S., \& Scherer, D.G. (1998). Female juvenile delinquency: misunderstood by the juvenile justice system, neglected by social science. Laws and human behavior, 22, 81-107.

Hughes, H.M., Parkinson, D., \& Vargo, M. (1989). Witnessing spouse abuse and experiencing physical abuse: a «double whammy»? Journal of Family Violence, 4, 197-209.

Ireland, T., \& Widom, C.S. (1994). Childhood victimization and risk for alcohol and drug arrests. International Journal of the Addictions, 29, 235-274.

Ireland, T.O., Smith, C.A., \& Thornberry, T.P. (2002). Developmental issues in the impact of child maltreatment on later delinquency and drug use : reconsidering the strength and boundaries of the relationship. Criminology, 40, 359-400.

Kakar, S. (1996). Child abuse and delinquency. New York: University Press of America, Inc.

Kandel, D., Chen, K., \& Gill, A. (1995). The impact of drug use on earnings: a life-span perspective. Social Forces, 74, 243-270. 
Kang, S.Y., Magura, S., Laudet, A., \& Whitney, S. (1999). Adverse effect of child abuse victimization among substance-using women in treatment. Journal of Interpersonal Violence, 14, 657-670.

Kaplan, S.J., Pelcovitz, D., Salzinger, S., Weiner, M., Mandel, F.S., Lesser, M.L., \& Labruna, V.E. (1998). Adolescent physical abuse : risk for adolescent psychiatric disorders. American Journal of Psychiatry, 155, 954-959.

Kaplan, S.J., Pelcovitz, D., \& Labruna, V.E. (1999). Child and adolescent abuse and neglect research: a review of the past 10 years. Part 1: Physical and emotional abuse and neglect. Journal of the American academy of child and adolescent psychiatry, 38, 1214-1222.

Keenan, K., Loeber, R., \& Green, S. (1999). Conduct disorder in girls : a review of the literature. Clinical Child and Family Psychology Review, 2, 3-19.

Kendall-Tackett, K.A., Williams, L.M., \& Finkelhor, D. (1993). Impact of sexual abuse on children : a review and synthesis of recent empirical studies. Psychological Bulletin, 113, 164-180.

Kendler, K.S., Bulik, C.M., Silberg, J., Hettema, J.M., Myers, J., \& Prescott, C.A. (2000). Childhood sexual abuse and adult psychiatric and substance use disorders in women: an epidemiological and cotwin control analysis. Archives of general psychiatry, 57, 953-959.

Kolbo, J.R., Blakely, E.H., \& Engleman, D. (1996). Children who witness domestic violence: a review of empirical literature. Journal of Interpersonal Violence, 11, 281-293.

Langhinrichsen-Rohling, J., Lewinsohn, P., Rohde, P., Seeley, J., Monson, C.M., Meyer, K.A., \& Langford, R. (1998). Gender differences in the suiciderelated behaviors of adolescents and young adults. Sex Roles, 39, 839-851.

Lemmon, J.H. (1999). How child maltreatment affects dimensions of juvenile delinquency in a cohort of low-income urban youths. Justice Quarterly, 16, 357-376.

Locke, T.F. \& Newcomb, M.D. (2004). Child maltreatment, parent alcohol- and drug-related problems, polydrug problems, and parenting practices: a test of gender differences and four theoretical perspectives. Journal of Family Psychology, 18, 120-134.

Manly, J.T., Cicchetti, D., \& Barnett, D. (1994). The impact of subtype, frequency, chronicity, and severity of child maltreatment on social competence and behavior problems. Development and Psychopathology, 6, 121-143.

Mannarino, A.P., Cohen, J.A., Smith, J.A., \& Moore-Motily, S. (1991). Six and twelve-month follow-up of sexually abused girls. Journal of Interpersonal Violence, 6, 494-511.

McClellan, D.S., Farabee, D., \& Crouch, B.M. (1997). Early victimization, drug use and criminality: a comparison of male and female prisoners. Criminal Justice and Behavior, 24, 455-476.

McCloskey, L.A., Figueredo, A.J., \& Koss, M.P. (1995). The effects of systemic family violence on children's mental health. Child Development, 66, 12391261.

McCloskey, L.A. (1997). Continuum of harm : sexual abuse of girls and women across the lifespan. In D. Cicchetti \& S.L. Toth (eds), Developmental perspec- 
tives on trauma: theory, research, and intervention: Rochester symposium on developmental psychopathology, vol. 8. Rochester, NY: Rochester University Press.

McCloskey, L.A., \& Lichter, E.L. (2003). The contribution of marital violence to adolescent aggression across different relationships. Journal of Interpersonal Violence, 18, 390-412.

McGee, R.A., Wolfe, D.A., \& Wilson, S.K. (1997). Multiple maltreatment experiences and adolescent behavior problems: adolescents' perspectives. Development and Psychopathology, 9, 131-149.

Merrill, L.L., Guimond, J.M., Thomsen, C.J., \& Milner, J.S. (2003). Child sexual abuse and number of sexual partners in young women: the role of abuse severity, coping style, and sexual functioning. Journal of Consulting and Clinical Psychology, 71, 987-996.

Moffitt, T.E., Caspi, A., Rutter, M., \& Silva, P.A. (2001). Sex differences in antisocial behavior: conduct disorder, delinquency and violence in the Dunedin Longitudinal Study. New York: Cambridge University Press.

Morrill, A.C., Kasten, L., Urato, M., \& Larson, M.J. (2001). Abuse, addiction and depression as pathways to sexual risk in women and men with a history of substance abuse. Journal of Substance Abuse, 13, 169-184.

Newcomb, M.D., \& Bentler, P.M. (1988). Impact of adolescent drug use and social support on problems of young adults : a longitudinal study. Journal of Abnormal Psychology, 97, 64-75.

Osofsky, J.D. (1999). The impact of violence on children. Future of Children, 9, 33-49.

Paolucci, E.O., Genuis, M., \& Violato, C. (2001). A meta-analysis of the published research on the effects of child sexual abuse. Journal of Psychology, 135, 17-36.

Pears, K.C., \& Capaldi, D.M. (2001). Intergenerational transmission of abuse: a two-generational prospective study of an at-risk sample. Child abuse and neglect, 25, 1439-1461.

Putnam, F.W. (2003). Ten-year research update review : child sexual abuse. Journal of the American academy of child and adolescent psychiatry, 42, 269-278.

Radloff, L. (1977). The CES-D Scale : a self-report depression scale for research in the general population. Applied psychological measurement, 1, 385-401.

Riggs, D.S., O’Leary, K.D., \& Breslin, F.C. (1990). Multiple correlates of physical aggression in dating couples. Journal of Interpersonal Violence, 5, 61-73.

Roberts, R., O'Connor, T., Dunn, J., Golding, J., \& The ALSPAC Study Team. (2004). The effects of child sexual abuse in later family life; mental health, parenting and adjustment of offspring. Child abuse and neglect, 28, 525-545.

Rossman, B.B.R. (2001). Longer term effects of children's exposure to domestic violence. In S.A. Graham-Bermann \& J.L. Edelson (eds), Domestic violence in the lives of children. Washington, DC: American Psychological Association.

Rutter, M., Caspi, A., \& Moffitt, T.E. (2003). Using sex differences in psychopathology to study causal mechanisms: unifying issues and research strategies. Journal of Child Psychology and Psychiatry and Allied Disciplines, 44 (8), 1092-1115. 
Salzinger, S. (1999). Determinants of abuse and the effects of violence on children and adolescents. In A.J. Goreczny \& M. Hersen (eds), Handbook of pediatric and adolescent health psychology. Boston, MA: Allyn et Bacon, Inc.

Sampson, R., \& Laub, J. (1993). Crime in the making: patbways and turning points through life. Cambridge, MA: Harvard University Press.

Siegel, J.A., \& Williams, L.M. (2003). The relationship between child sexual abuse and female delinquency and crime: a prospective study. Journal of Research in Crime and Delinquency, 40, 71-94.

Silverman, A.B., Reinherz, H.Z., \& Giaconia, R.M. (1996). The long-term sequelae of child and adolescent abuse: a longitudinal community study. Child abuse and neglect, 20, 709-723.

Simons, R.L., Wu, C., Johnson, C., \& Conger, R. (1995). A test of various perspectives on the intergenerational transmission of domestic violence. Criminology, 33, 141-171.

Simpson, T.L., \& Miller, W.R. (2002). Concomitance between childhood sexual and physical abuse and substance use problems. Clinical Psychology Review, 22, 27-77.

Simpson, T.L. (2003). Childhood sexual abuse, PTSD, and the functional roles of alcohol use among women drinkers. Substance use and misuse, 38, 249-270.

Smith, C.A., \& Thornberry, T.P. (1995). The relationship between childhood maltreatment and adolescent involvement in delinquency. Criminology, 33, 451-481.

Smith, C.A., Ireland, T.O., \& Thornberry, T.P. (2004). Adolescent maltreatment and its impact on young adult antisocial behavior. Manuscrit non publié.

Spohn, R.E. (2000). Gender differences in the effect of child maltreatment on criminal activity over the life course. In G.L. Fox \& M.L. Benson (eds), Contemporary perspectives in family research: families, crime and criminal justice. New York: Elsevier Science.

Stith, S., Rosen, K.H., Middleton, K., Busch, A.L., Lundeberg, K., \& Carlton, R.P. (2000). The intergenerational transmission of spouse abuse: a metaanalysis. Journal of Marriage and the Family, 62, 640-654.

Straus, M.A., \& Gelles, R.J. (1990). Pbysical violence in American families : risk factors and adaptations to violence in 8,145 families. New Brunswick, NJ: Transaction.

Survey Research Laboratory, University of Illinois (1987). Chicago area general population survey on AIDS : screening questionnaire. Chicago: University of Illinois Survey Research Laboratory.

Swanston, H.Y., Parkinson, P.N., O’Toole, B.I., Plunkett, A.M., Shrimpton, S., \& Oates, R.K. (2003). Juvenile crime, aggression and delinquency after sexual abuse. British Journal of Criminology, 43, 729-749.

Thompson, M.P., Kingree, J.B., \& Desal, S. (2004). Gender differences in long-term health consequences of physical abuse of children: data from a nationally representative survey. American Journal of Public Health, 94, 599-604.

Thornberry, T.P., Bjerregaard, B., \& Miles, W. (1993). The consequences of respondent attrition in panel studies: a simulation based on the Rochester Youth Development Study. Journal of Quantitative Criminology, 9, 127-158. 
Thornberry, T.P., Ireland, T.O., \& Smith, C.A. (2001). The importance of timing: the varying impact of childhood and adolescent maltreatment on multiple problem outcomes. Development and Psychopathology, 13, 957-979.

Thornberry, T.P., Krohn, M.D., Lizotte, A.J., Smith, C.A., \& Tobin, K. (2003). Gangs and delinquency in developmental perspective. New York: Cambridge University Press.

Thornberry, T.P., \& Krohn, M.D. (sous presse). Applying interactional theory to the explanation of continuity and change in antisocial behavior. In D.P. Farrington (ed.), Integrated developmental and life course theories of offending: advances in criminological theory, vol. 14. Piscataway, NJ: Transaction.

Toth, S.L., Manly, J.T., \& Cicchetti, D. (1992). Child maltreatment and vulnerability to depression. Development and Psychopathology, 4, 97-112.

Trickett, P.K., Reiffman, A., Horowitz, L.A., \& Putnam, F.W. (1997). Characteristics of sexual abuse trauma and the prediction of developmental outcomes. In D. Cicchetti \& S.L. Toth (eds), Developmental perspectives on trauma: theory, research, and intervention: Rochester symposium on developmental psychopathology, vol 8. Rochester, NY: University of Rochester Press.

USDHHS (U.S. Department of Health and Human Services) (2004). Child Maltreatment 2002. Washington, DC: Administration for Children and Families, Administration on Children, Youth and Families. <http://www.acf.hhs. gov/programs/cb/publications/cm02/cm02.pdf $>$.

White, H.R., \& Widom, C.S. (2003). Intimate partner violence among abused and neglected children in young adulthood: the mediating effects of early aggression, antisocial personality, hostility, and alcohol problems. Aggressive behavior, 29, 332-345.

Widom, C.S. (1989a). The intergenerational transmission of violence. New York: Harry Frank Guggenheim Foundation.

Widom, C.S. (1989b). Child abuse, neglect, and violent criminal behavior. Criminology, 27, 251-271.

Widom, C.S., Ireland, T., \& Glynn, P. (1995). Alcohol abuse in abused and neglected children followed-up: are they at increased risk? Journal of Studies on Alcobol, 56, 207-217.

Widom, C.S., \& Kuhns, J. (1996). Childhood victimization and subsequent risk for promiscuity, prostitution, and teenage pregnancy: a prospective study. American Journal of Public Health, 86, 1607-1612.

Widom, C.S., \& Shepard, R. (1996). Accuracy of adult recollections of childhood victimization: Part 1. Childhood physical abuse. Psychological assessment, 8, 412-421.

Widom, C.S., \& White, H.R. (1997). Problem behaviours in abused and neglected children grown up: Prevalence and co-occurrence of substance abuse, crime and violence. Criminal behaviour and mental bealth, 7, 287-310.

Widom, C.S. (1998). Childhood victimization: early adversity and subsequent psychopathology. In B.P. Dohrenwend (ed.), Adversity, stress and psychopathology. New York: Oxford University Press. 
Widom, C.S., Weiler, B.L., \& Cottler, L.B. (1999). Childhood victimization and drug abuse: a comparison of prospective and retrospective findings. Journal of Consulting and Clinical Psychology, 67, 867-880.

Widom, C.S. (2000a). Child abuse and later effects. National Institute of Justice journal, January, 2-9.

Widom, C.S. (2000b). Understanding the consequences of childhood victimization. In R.M. Reece (ed.), Treatment of child abuse. Baltimore, MD: John Hopkins University Press.

Widom, C.S., \& Hiller-Sturmhofel, S. (2001). Alcohol abuse as a risk factor for and consequence of child abuse. Alcohol research and health, 25, 52-57.

Widom, C.S., \& Maxfield, M.G. (2001). An update on the "Cycle of Violence». Washington, DC: National Institute of Justice.

Wolfe, D.A., Wekerle, C., Reitzel-Jaffe, D., \& Lefebvre, L. (1998). Factors associated with abusive relationships among maltreated and nonmaltreated youth. Development and psychopathology, 10, 61-85.

Wolfe, D.A., Scott, K., Wekerle, C., \& Pittman, A. (2001). Child maltreatment: risk of adjustment problems and dating violence in adolescence. Journal of the American Academy of Child and Adolescent Psychiatry, 40, 282-289. 